\title{
3D Topographic Data Modelling: Why Rigidity Is Preferable to Pragmatism
}

\author{
Friso Penninga \\ Delft University of Technology, OTB, section GIS Technology, \\ Jaffalaan 9, 2628 BX Delft, The Netherlands \\ F.Penninga@otb.tudelft.nl
}

\begin{abstract}
In this paper two concepts for modelling 3D topography are introduced. The first concept is a very pragmatic approach of 3D modelling, trying to model as much as possible in (less complicated) 2.5D and use 3D modelling only in exceptional cases. The idea is to use a constrained TIN in 2.5D and place 3D TENs on top or below this surface. As both data structures use the same simplexes (nodes, edges, triangles) this integration should be very well possible. At a conceptual level this approach seems suitable, but at design level serious problems occur. To overcome these a rigid approach is developed, modelling all features in a 3D TEN, including the air above and earth beneath these topographic features. This model is stored and maintained within a spatial database. Despite its more advanced concept, it is shown that this approach offers huge advantages compared to the initial pragmatic approach.
\end{abstract}

\section{Introduction}

This paper describes two modelling concepts developed within the research on 3D Topography as carried out within the GIS Technology group at Delft University of Technology. Initially the aim was a pragmatic approach, in which applicability was one of the keywords. However, during the research we came to the conclusion that our pragmatic approach seemed suitable at conceptual level, but that it causes some serious modelling problems at design level. Based on the identified strengths of this initial model a rigid approach is developed, which turned out to actually simplify most of our problems at design level, although it is more advanced at conceptual level.

This paper starts with an introduction on the backgrounds of the 3D Topography research in Paragraph 2, including a short overview of relevant available data sets. Next the concepts of the initial pragmatic modelling approach are introduced in Paragraph 3. Its drawbacks are addressed and some preliminary conclusions on the initial modelling approach described. Based on these conclusions the concepts of the new rigid approach are presented in Paragraph 4, followed by some remarks on the implementation of this approach. The paper ends with final conclusions and future research in Paragraph 5. 


\section{Backgrounds of the 3D Topography Research}

\subsection{The Need for the Third Dimension}

Most current topographic products are limited to representing the real world in only two dimensions. As the real world exists of three dimensional objects, which are becoming more and more complex due to increasing multiple land use, accurate topographic models have to cope with the third dimension. The overall goal of this research is to extend current topographic modelling into the third dimension. Applications of 3D modelling are not limited to the terrain surface and objects built directly on top or beneath it, as geological features and air traffic or telecommunication corridors can be modelled too.

Most initiatives on developing 3D GIS focus on supporting visualisation, often in Virtual Reality-like environments. One of the objectives of this 3D modelling research is to enable 3D analysis as well, as this traditional GIS-strength lacks until now in most 3D GIS approaches. Another important assumption within this research follows from the required wide variety of applications of topographic data. As topography is ranked high in the spatial data infrastructure hierarchy, one cannot optimize the data model for one specific purpose. One has to be able to serve the complete range of user applications, regardless whether these applications require for instance optimal visualisation capabilities or optimal analytical capabilities.

In 3D modelling one needs a 3D primitive (a volume) beside points, lines and faces to represent 3D objects accurately. Earlier research proposed amongst others using simplexes (point, line, triangle, tetrahedron) (Carlson 1987), points, lines, surfaces and bodies (3D Formal Data Structure (FDS)) (Molenaar 1990a, Molenaar 1990b, Molenaar 1992), combining Constructive Solid Geometry (CSG) and a B-rep. (de Cambray 1993) and integrating a 2.5D Triangulated Irregular Network (TIN) with 3D FDS (Pilouk 1996). In applications polyhedrons are often used as 3D primitive (Zlatanova 2000, Stoter 2004). These publications on 3D modelling concepts are often limited to a conceptual description of the use of a 3D primitive, without addressing any of the actual problems concerning the use of these 3D primitives as in analysis. As a result true implementations (besides some very small experiments) are rare, thus not proving actual usefulness of the concepts.

\subsection{Current Available Data Sets}

The initial approach to extend topographic modelling into 3D is both supply and demand driven. The required data sets -both topography and height databecome available in a growing number of countries. 2D Topographic data sets are available and are being converted into object-oriented models, as this offers huge advantages in digital processes such as GIS analysis. Due to the growing popularity of airborne laser scanning high resolution height data becomes available. Combining both types of data can lead to full $3 \mathrm{D}$ data. Within the presented research Dutch data sets will be used in implementation tests. The 
height information is available in a Digital Elevation Model (DEM) called the AHN (Actual Height model the Netherlands). As large parts of the Netherlands are situated below sea level accurate large scale height data is of great importance, thus leading to the development of the AHN, a height model with one height point for on average every $16 \mathrm{~m} 2$. The most relevant topographic data set (1:10.000) is currently being converted by the Dutch Topographic Survey into an object-oriented structure called TOP10NL.

At the demand side developments as increasing multiple land use and rising awareness of the importance of sustainable urban development (both caused by urban space scarcity), increase the need for real 3D topographic data sets. At dataset level the major shortcomings of the current 2D products lie in the absence of height information for buildings and other constructions, which is essential for for instance noise and odour modelling and in problems at viaducts with crossing (rail)roads on different levels.

\section{Initial Modelling Concept}

Based on the shortcomings at product level of the traditional topographic map the initial idea was that only in some specific cases true 3D modelling would be necessary, whereas in the majority of cases modelling in $2.5 \mathrm{D}$ would be sufficient. I define $2.5 \mathrm{D}$ modelling as using a single height value at every $\mathrm{x}, \mathrm{y}$-coordinate. Sometimes this is referred to as 'strict 2.5D modelling', as some people define 2.5 modelling as using 2D-simplexes (faces) in 3D space (Pilouk 1996), thus enabling several height values on one $\mathrm{x}, \mathrm{y}$-coordinate.

As $2.5 \mathrm{D}$ modelling is far less complex than 3D modelling, this has lead to the concept of combined 2.5D/3D modelling. The basic assumption is that the earth's surface can be modelled in 2.5D and that some more complex situations like buildings, viaducts or tunnels can be placed on top or below this surface. Apart from the intention to extend topographic models from 2D into 3D another important characteristic of the new modelling approach is to introduce the use of a foundation data structure. Within a data structure redundant data storage (geometry) can be avoided and the relationships between objects enable validation. In $2 \mathrm{D}$ one might require for instance that all objects form a planar partition, thus banning empty spaces between objects. The availability of topological relationships can also improve query performance during analyses.

\subsection{Concepts of the Integrated TIN/TEN Approach}

This leads to the concept of a topographic terrain representation in an integrated TIN/TEN model (TIN: Triangulated Irregular Network / TEN: Tetrahedronized Irregular Network). Four types of topographic features can be determined: 0D (point features), 1D (line features), 2D (area features) and 3D (volume features). For each type of feature simplexes of corresponding dimension are available to represent the features with, i.e. nodes, edges, triangles and tetrahedrons. A great advantage of using these simplexes is the well-defined character of the mutual 
relationships: a $\mathrm{kD}$ simplex is bounded by $\mathrm{k}+1$ ( $\mathrm{k}-1) \mathrm{D}$-simplexes (Pilouk 1996). This means that for instance a 2D simplex (a triangle) is bounded by three 1D simplexes (edges) and a 3D simplex (tetrahedron) is bounded by four 2D simplexes (triangles). The second important advantage of simplexes is the flatness of the faces, which enables one to describe a face using only three points. The third advantage is that every simplex, regardless its dimension, is convex, thus making convexity testing unnecessary. This quality simplifies point-in-polygon test significantly. The price for this comes with increased modelling complexity. Compared to for instance using polyhedrons as 3D primitive it will be clear that there exists a 1:1 relationship between a 3D feature (for instance a building) and its representation (the polyhedron), but that there will be a 1:n relationship between this 3D feature and its tetrahedrons. However, as long as one is able to hide this complexity from the average user, the advantages will overcome this drawback. To further illustrate the strength of using well-defined primitives, consider a real estate tax application that determines the tax assessment based on the volume of the building. In order to automate this process, a formula for determining volumes is required. Designing a formula capable of determining a polyhedron's volume is more complex due to the unlimited variation in shape. Contrarily, implementing a formula for the volume of a tetrahedron is straightforward, it only has to be applied several times as a building will be represented as a set of tetrahedrons. This repetition is however exactly what computers are good for.

The concept of the integrated TIN/TEN model is to represent 0D-2D objects in a TIN and 3D objects as separate TENs, that will be placed on top or below the TIN. This principle is illustrated in Figure1, Note that the TIN for simplicity reasons is shown as a $2 \mathrm{D}$ TIN but that the model uses a 2.5D TIN. As both TINs and TENs are using triangles they can be 'put together' by making sure that they both contain the corresponding triangles.

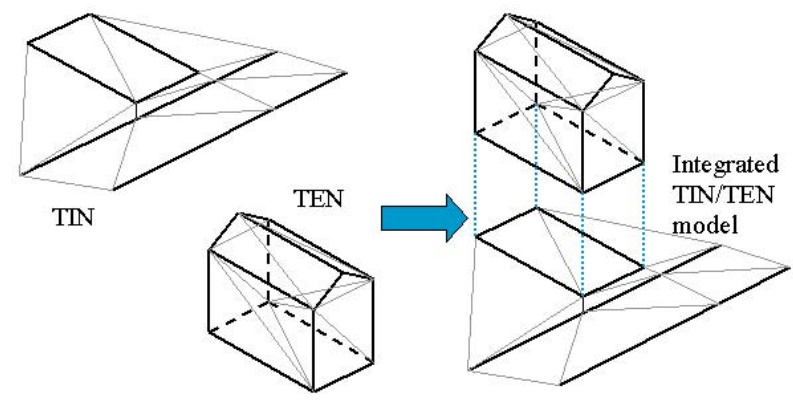

Fig. 1. Principle of modelling in an integrated TIN/TEN model

The underlying data structure can be designed quite straightforward, as the model only consists of nodes, edges, triangles and tetrahedrons. In Figure 2 an initial UML diagram is given of the structure. It consists of the four primitives, 
linked as described by the definition of Pilouk: a $\mathrm{kD}$ primitive is bounded by $\mathrm{k}+1(\mathrm{k}-1) \mathrm{D}$ primitives. Flags (booleans) indicate whether a node/edge/triangle is part of the TIN, TEN or both. However, it shows that in this simple structure some problems occur as some attributes apply only on TINs or on TENs. Furthermore these attributes are in fact associations and have to be modelled accordingly. For example: within a TIN an edge has two neighbouring faces (left/right), within a TEN the number of associated faces in unbounded. An issue that will not be addressed in this section is whether to store only the node geometry and compute all other geometries based on the these nodes or to store the geometry for every simplex, as it is currently modelled in the class diagram.

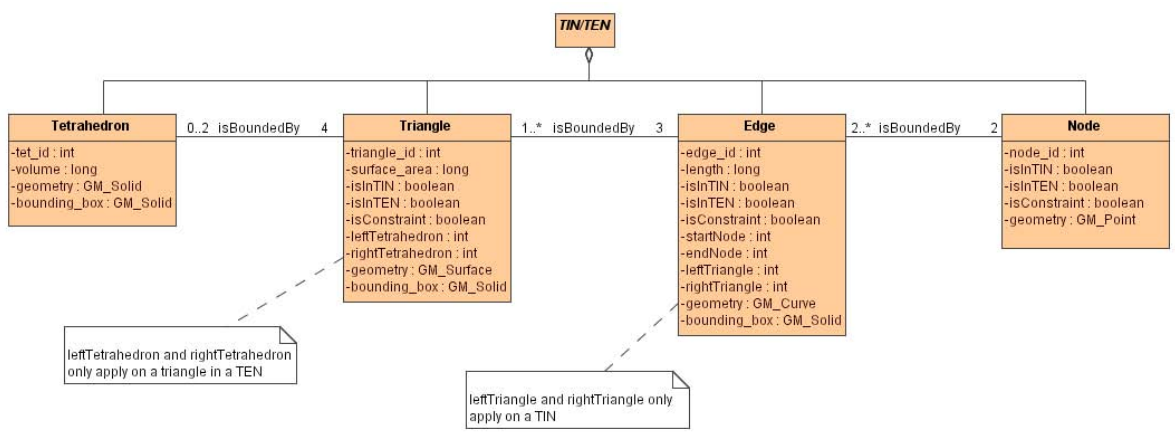

Fig. 2. Initial UML class diagram of the data structure. Note that some UML class diagram modelling rules are ignored in this diagram.

The attempt of the first conceptual model to store and integrate TIN and TENs at the most fundamental level was futile. Conceptually a TIN edge is not the same as a TEN edge and the same holds for TIN triangles and TEN triangles. Its geometries might be identical, but TIN and TEN edges and triangles have different mutual relationships. Therefore it is necessary to model the TIN and TEN separately (and where appropriate link or even merge its components). However a close relationship exists between for instance a TIN edge and a TEN edge, as they both are 1-simplexes. The UML class diagram in Figure 3 illustrates this relationship explicitly. An optional 'isEquivalentTo' relationship is defined between TIN and TEN nodes, edges and triangles. Note that this relation could indicate redundancy in the model, depending the actual implementation at storage level. This relationship is also visible in Figure 1 where the floor of the building is modelled in the TEN with nodes, edges and faces, equivalent to the nodes, edges and faces in the footprint in the TIN. Parallel to the Formal Data Structure (Molenaar 1990a) the initial modelling approach is feature-oriented. The model is able to represent point, line, area and volume features. In order to integrate TIN and TEN (and thus the 2.5D world with the $3 \mathrm{D}$ world) also semantically, the footprint of a volume feature is also integrated in the TIN. As a result the TIN can be considered to form a representation at 


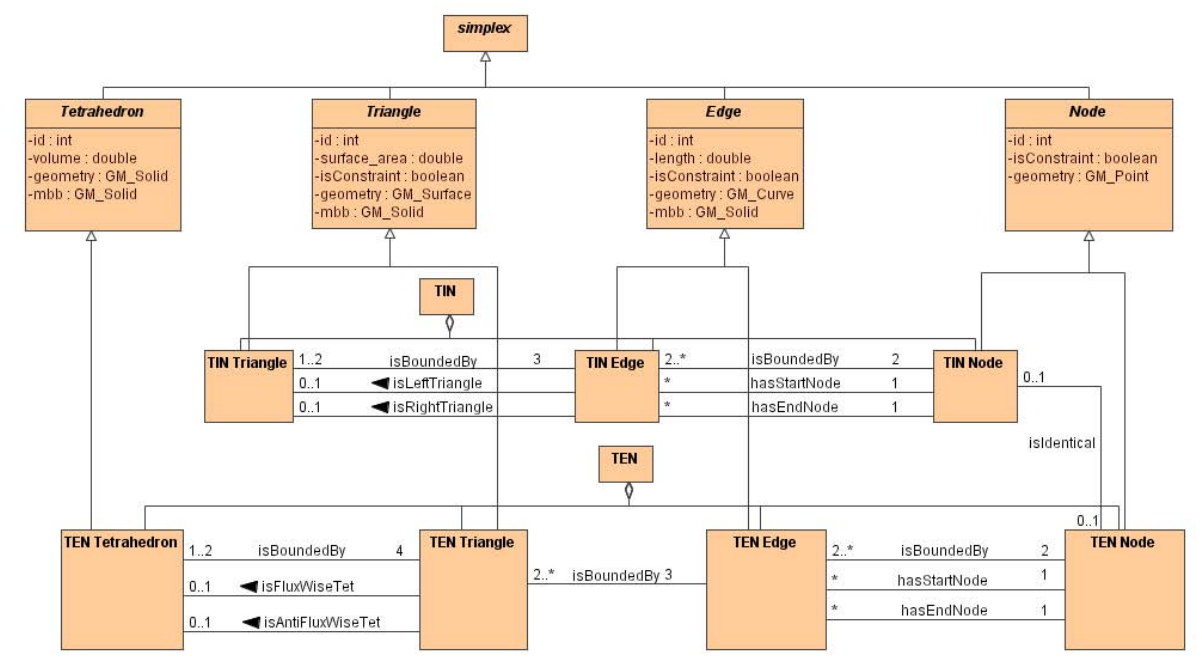

Fig. 3. UML class diagram of the integrated TIN/TEN model

terrain level. Figure 4 integrates the features in the UML class diagram. In this figure the connection between the TIN and the TEN at data structure level is only available at node level (and not at edge or triangle level); the reasons for this will be clarified in the next section. Three different types of linking can be distinguished:

- link at triangle level (thus 'glueing' the TENs on top of the TIN)

- link at edge level (thus 'stitching' the TENs on top of the TIN)

- link at node level (thus 'nailing' the TENs on top of the TIN)

Linking at the node level is the most fundamental one of this three, as edges and (indirect) triangles are defined by their nodes. However in order to optimize analytical capabilities one would prefer to have the link available at triangle level (which implies also the relationship on edge and node level).

\subsection{Drawbacks Initial Modelling Concept}

Although our initial modelling approach seems to make sense from a practical topographic point in a UML class diagram, it has some serious hidden problems. The first problem lies within the integration of the TIN with the TENs. At a conceptual level the 'isEquivalentTo' relationship is an appropriate way of linking both models. However, in order to optimize analytical capabilities one would prefer this link to exist on node as well as on edge and triangle level. In order to do so, one needs an implementation that takes care of ensuring the 1:1 relationship between TIN surface and TEN bottom. If one considers the example of a building placed on top of the terrain (as illustrated in Figure 1), not only the footprint of the building should match in TIN and TEN, but also the internal 


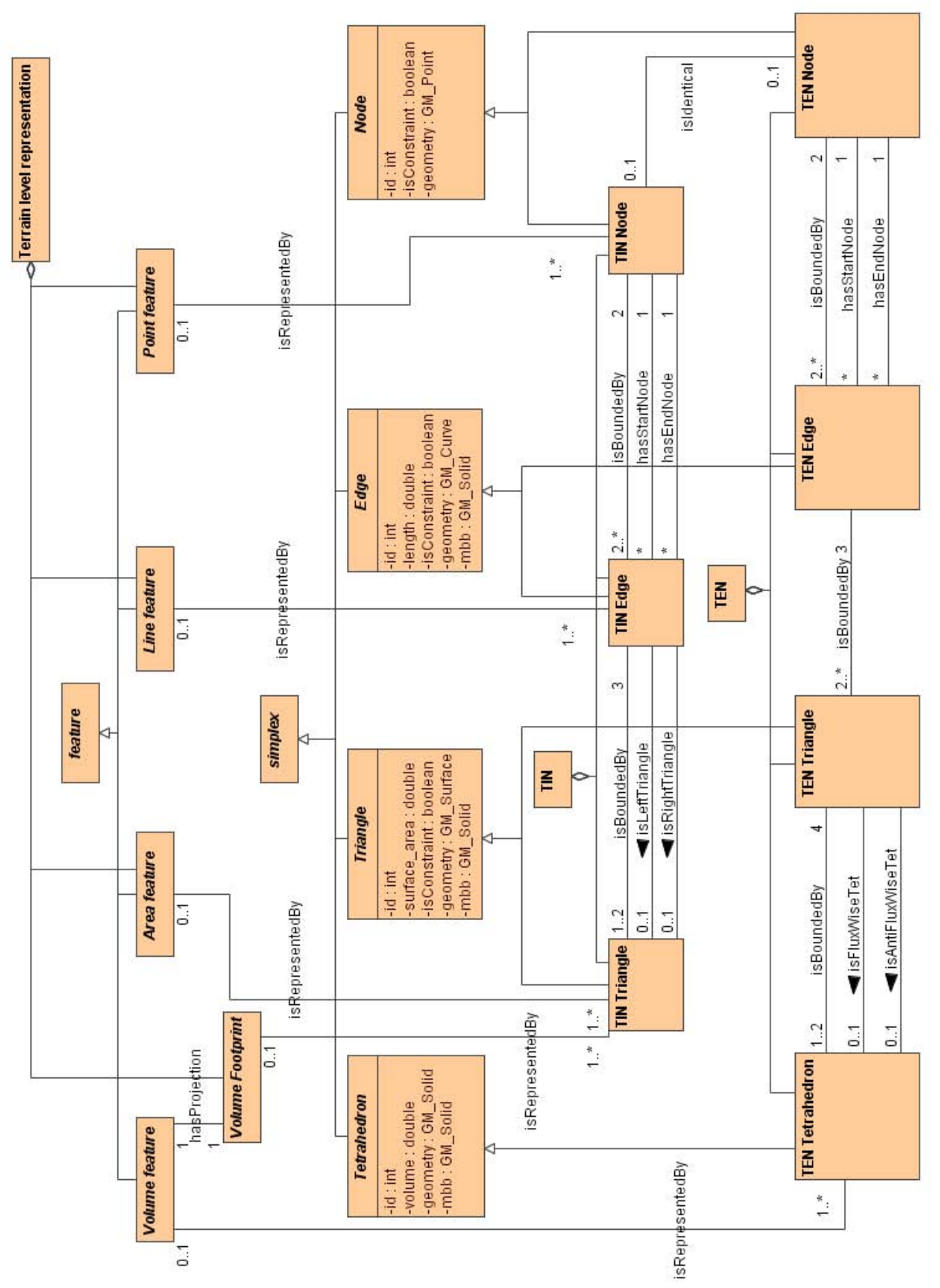

Fig. 4. UML class diagram of the feature-based integrated TIN/TEN model

edges in the shared face. The building will be represented as a rectangle in the TIN and this rectangle is identical to the floor boundaries in the TEN. However, in order to ensure the 1:1 relationship also on edge and face level, one needs the 
guarantee that the internal edge that triangulates this rectangle is the same diagonal in both data structures. Unfortunately it is not possible to ensure a match between the TIN and TEN triangles, as constrained triangulations and tetrahedronizations are only capable of handling constrained edges (Shewchuk 2004). This implies that not only the outer boundaries should be handled as constraints, but also this internal edge. As a result the outcome of the TIN triangulation can be transferred as input into the TEN triangulation or vice versa. This dependency wouldn't be a problem if one could be sure that these constraints will be triangulated without problems. However additional Steiner points are often required in order to enable tetrahedronization (as Schönhardts polyhedron (Schönhardt 1928) can’t be tetrahedronized without additional Steiner points) or to improve the quality of a constrained Delaunay triangulation (and thus the numerical stability (Shewchuk 1997)). As Steiner points are inserted for instance in the TIN, they need to be transferred into the TEN, accompanied with the additional edges created by the Steiner points. At the same time the TEN algorithm might insert additional Steiner points, which should be transferred back to the TIN, thus resulting in the threat of a never-ending exchange of Steiner points.

This problem can probably be solved (partially) by handling Steiner points in a different way. Within algorithms used in GIS Steiner points are almost always used to split long edges into smaller ones. In the more general research field of meshing, where amongst others triangulation and tetrahedronization are used in order to simplify complex objects to enable appliance of partial differential equations, Steiner points are also added in the interior of a triangle or tetrahedron (Shewchuk 1997). In particular most refinement algorithms select skinny triangles and add the centrepoint of the circumcircle as a Steiner point. In TENs the centrepoint of the circumsphere can be used. Within GIS adding internal nodes is rather unusual, probably due to the fact that this data is collected by surveying techniques as GPS or photogrammetry, which are point measurements. As a result a node usually represents a measurement, which is not the case for Steiner points in the interior of an object. However, the question whether every polyhedron can be triangulated or tetrahedronized without adding Steiner points at the boundary of the object is theoretically not answered yet.

Although the modelling approach is quite straightforward ("model in 2.5D, only in exceptional cases model in 3D"), the questions how to link both models and when to switch between the two representations are not easy to answer (and thus to implement in practice). An important design question is whether both TIN and TEN should exist in case of more complex situations or that only the TEN should be available in these situations (implying a 'hole' in the TIN), thus creating 2.5D representation that is not a surface partition. Both approaches (TIN+TEN and TIN-TEN) are suitable in certain situations. One can imagine a situation in which a single building is represented in a TEN. At this location it will be quite easy to include the footprint in the TIN, thus using a TIN+TEN approach. However, if one considers the complex situation in Figure 5, in which a highway and railroad tracks are planned in tunnels, with a station and offices 


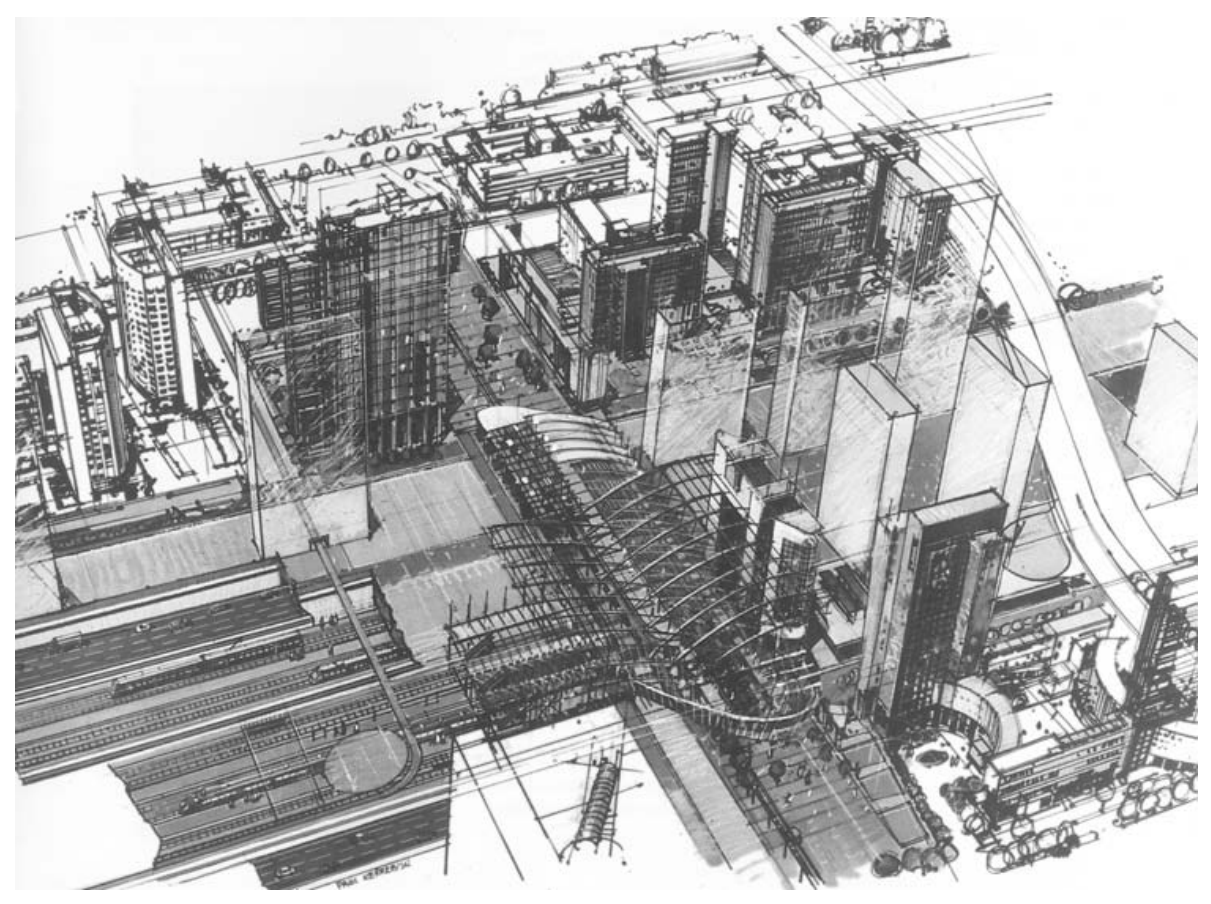

Fig. 5. Impression of the plans for the renewal of Amsterdam WTC Station, with several tunnels, offices and a station build on top of each other

build on top, the question is how to give a meaningfull definition of the 'terrain level' and thus what has to be included in the $2.5 \mathrm{D}$ representation. In such a situation it would make more sense to model the entire complex situation in one TEN, which shares its borders with the surrounding TIN, thus using a TIN-TEN approach. Virtual closure surfaces (Kolbe, Gröger \& Plümer 2005) can be added to obtain a closed surface, although these surfaces have no relationships with actual features.

As a result from both examples one cannot select either the TIN+TEN or the TIN-TEN approach alone. It makes sense to use both approaches, but how to satisfactory define a general rule when to apply TIN+TEN and when TIN-TEN? This criterion adds more complexity to the initial simple modelling concept. In order to further contribute to the confusion, let's concentrate at the question which feature types are modelled in the TIN and which in a TEN. If one considers the simplified viaduct in Figure 6, applying the initial modelling approach would imply that only the viaduct itself will be modelled in $3 \mathrm{D}$ and the ascent and descent in 2.5D. Suppose that both on and under the viaduct a highway exists. In this case the bottom highway is represented in a TIN and the upper highway in a TEN. As a result the upper highway will have a thickness, while the bottom highway has not. One can also decide to only label the top triangles of the TEN as highway, but this will result in a meaningless volume, acting as a 'carrier object' 


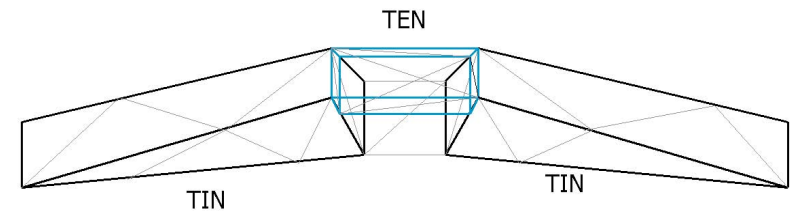

Fig. 6. Simplified viaduct, partially modelled as TEN

for the highway object. Regardless the chosen approach, it will still be an issue how to define the thickness of the TEN, as this data will not be available. One could use some kind of standard thickness, based on prior knowledge of some civil engineering rules of thumb. Still, why should one include this thickness or a 'carrier object' for highways on a viaduct and not include the standard thickness of the foundation of the bottom highway? This inconsistency is hard to accept.

The earlier observation that it will be difficult to define a $2.5 \mathrm{D}$ terrain surface everywhere implies that it is apparently not possible to extend all characteristics of a $2 \mathrm{D}$ representation into $2.5 \mathrm{D}$, especially when this $2.5 \mathrm{D}$ representation has to fit with 3D TENs. In 2D a topographic representation can be considered as a topologically closed surface. This plays an important role in consistency checks of the data. However in 2.5D this rule no longer applies, for instance if one thinks of a tunnel entrance. In $2 \mathrm{D}$ the road stops at the tunnel entrance, which is also the border of the terrain feature lying above the tunnel. In $2.5 \mathrm{D}$ there will be a vertical gap between these two features, resulting in a non-watertight surface.

So far it is clear that - after solving a number of practical problems as described above - it will be possible to create a model in which TIN and TENs are combined, thus enabling a representation in which both surface and volume objects are present. Now the question arises whether this representation also offers the required functionality. For instance, Figure 6 illustrated that it will be possible to model a viaduct as a combination of a TIN and a TEN, but does this model enable the user to analyse the clearance under the viaduct? In a geometrical sense a relationship can be discovered between the TIN triangle of the bottom highway and the TEN lying above, but only after some serious calculations. Wouldn't it be better if the air between the viaduct and the underlying terrain was also modelled, thus enabling the user to calculate the height of these 'air' tetrahedrons in order to solve his query? The same holds for a tunnel. In the TIN+TEN case the tunnel TEN is only attached to the TIN at both tunnel entrances, but would this enable a quick analysis of which buildings are located on top of the tunnel? If the earth between the tunnel and the buildings is also modelled, this would simplify the query. Again this raises the question whether it is possible to satisfactory define a general rule which 'air' or 'earth' tetrahedrons should be included and which not.

\subsection{Conclusions on the Initial Modelling Approach}

Our initial modelling approach has some strengths and weaknesses. At a conceptual level the TIN and TEN have very close relationships and can be combined 
in an integrated data structure. The combination of TIN and TEN fits with the important observation that large parts of a country can be considered to be $2.5 \mathrm{D}$ and therefore there is no need for more complicated 3D modelling in these areas. However at the lower level problems occur regarding the actual integration and connection of the TIN and TEN models. Maintaining a 1:1 relationship between a TIN triangle and a TEN triangle is difficult due to the addition of Steiner points. Another problem in linking the TIN and TEN lies within the limited analytical capabilities of the combined model, as 'empty' space between the TIN and TEN is not present in the model. Therefore the link is only clearly present at the shared boundaries of TIN and TEN. Another problem is the non-existence of a topologically closed surface in $2.5 \mathrm{D}$ in the combined $2.5 \mathrm{D} / 3 \mathrm{D}$ model.

\section{Proposed New Modelling Approach}

As the initial modelling approach had several drawbacks, a better approach was looked for. However, it was intended to retain the strengths of the initial modelling approach. These identified strengths were the point of departure for further developments:

- triangulation in $2 \mathrm{D} / 2.5 \mathrm{D} / 3 \mathrm{D}$ is a powerful data structure and offers computational advantages

- triangles correspond to well defined (flat) faces

- triangulations are suitable for storing objects in several dimensions

- large parts of a country can be represented in $2.5 \mathrm{D}$ due to the absence of true $3 \mathrm{D}$ shapes

\subsection{Fundamental Concepts}

Besides the strengths from the initial modelling approach the new approach is based on two fundamental observations:

- The ISO 19101 Geographic information - Reference model defines a feature as an 'abstraction of real world phenomena'. These real world phenomena have by definition a volumetric shape. In modelling often a less-dimensional representation is used in order to simplify the real world. Fundamentally there are no such things as point, line or area features; there are only features with a point, line or area representation (at a certain level of abstraction/generalization).

- The real world can be considered to be a volume partition. A volume partition can be defined (analogously to a planar partition) as a set of nonoverlapping volumes that form a closed modelled space. As a consequence objects like 'air' or 'earth' are explicitly part of the real world and thus have to be modelled.

These two observations contain no shocking new insights; the first principle even goes back as long as to ancient cartography. However, combining both observations leads to an important method of treating less dimensional representations. 
Based on the first observation one might wonder whether less-dimensional representations are even allowed in the new modelling approach, for instance using a face instead of a volume. The answer is positive, but only in special cases. Looking at the real world one can see that the features that are represented by faces are actually marking a border between two volume objects. For instance an area labelled as 'forest' might still be represented as a face, as it represents not only 'forest' but also implicitly the earth's surface, thus marking the transition between 'air' above and 'earth' beneath the surface. A lot of common modelling approaches are in its ways to define objects actual some kind of boundary representations. As in $2 \mathrm{D}$ a building is often represented by its walls; these walls actually mark the transition from the building's interior and the outside world. In the new modelling approach these volumes play a central role. The faces marking the borders between volumes might still be labelled, for instance as 'wall' or 'roof', but semantically they do not bound the building anymore, as the building in itself is represented by a volume, with neighbouring volumes that represent air, earth or perhaps another adjacent building.

At this point it might seem that also modelling 'air' and 'earth' in addition to all common topographic features is a very rigid approach of modelling, more serving the abstract goal of 'clean' modelling than an actual useful goal. This is however not the case. These air and earth objects do not just fill up the space between features of the other types, but are often also subject of analyses, such as noise and odour modelling. Another great advantage is the flexibility introduced by these features, as they enable future extensions of the data model. For instance, if one wants to model air traffic corridors, they can simply be inserted in the model. The space that is now simply labelled as 'air' can be subdivided in much more types, such as air traffic or telecommunication corridors. The same holds for the 'earth' tetrahedrons. In a later stage this general classification can be replaced by a more accurate one, for instance based on geotechnical and geological layers or polluted regions. Another advantage of modelling these 'empty' spaces is that it enables very pragmatic solutions for short term problems. If one thinks again of the viaduct in Figure 6, the problem was that feature instances of the same type were sometimes represented in $2.5 \mathrm{D}$ and sometimes in $3 \mathrm{D}$, even though the thickness was not known. As long as no real data is available at the viaduct, one might model both crossing roads as faces. The upper highway will be represented as a set of flat faces on the border of two volumes, this time both 'air' volumes. This might not be a desirable definite solution, but until the availability of real 3D data of viaducts this is a pragmatic solution. This 3D data will become available in the future as terrestrial laser scanning will become more and more common practice (see Figure 7 for a terrestrial laserscan of a bridge). As a last advantage the simplicity of the concept in itself can be mentioned: modelling 'everything' in a TEN is easier than modelling sometimes in a TIN, sometimes in a TEN. Immediately it should be mentioned that modelling in a TEN is more complex than modelling in a TIN, thus questioning whether modelling everything in a TEN will be also easier at implementation level. 


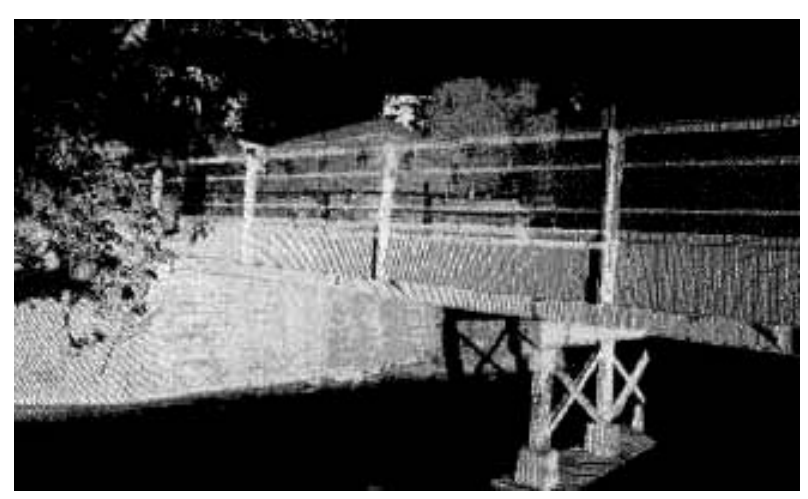

Fig. 7. Terrestrial laserscan of a bridge gives 3D information

Another interesting concept that is loosely related to the new modelling approach is an idea that still enables a user to work with a $2.5 \mathrm{D}$ TIN, even though the topographic model is stored as a full TEN. Up till now no distinction has been made between the way in which features are presented to the user and the way these representations are stored. One of the goals of the 3D Topography research is to implement the model in a spatial database, as this will improve manageability of the data. Databases have a rather nice feature and that is that one can work with views instead of tables. Whereas a table is physically stored in the database, a view can be seen as some kind of a virtual table. The user has all functionality as if he is working with a table, but the view is only a certain filter on top of one or more tables. Within our topography model one can think of node, edge, triangle and tetrahedron tables as basic storage structure of the 3D TEN. As stated at the beginning of this paragraph, the observation is that large parts of a country can be considered to be $2.5 \mathrm{D}$, thus it still would simplify some applications if a TIN surface would be available. The idea is now that it should be possible to define a view on top of the triangle table of the TEN in such a way that this view consist of TIN triangles. As seen in the previous paragraph linking the TIN with the TENs was very difficult due to several problems, amongst others with Steiner points. The concept of using a view on a TEN actually does not try to solve the integration problem of the TIN and TEN, but it actually avoids the problem completely. Using a view in queries and visualisation makes the user believe that there is a TIN, while it's actually just a subset of the TEN triangles. The idea is quite simple, but with all earlier integration problems in mind it is an ingenious solution. It is almost surrealistic, as on user level the TIN is available for all kind of applications, including navigating to neighbours via edges. It resembles (see Figure 8) a work of Rene Magritte, La Trahison des Images (the Betrayal of Images), with the caption 'Ceci n'est pas un pipe' (This is not a pipe). This is exactly what defining a view does with the average user: he is convinced that he is working with a TIN (in an analysis or visualisation), but actually there is no (physically stored) TIN, as he is presented with a subset of TEN triangles. 

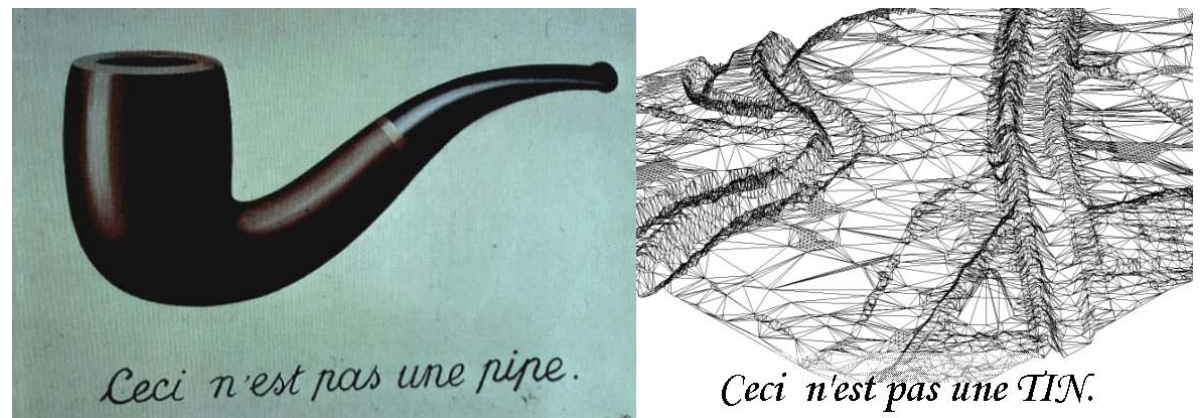

Fig. 8. La Trahison des Images, Rene Magritte, 1929 (left) and the TIN variant (right)

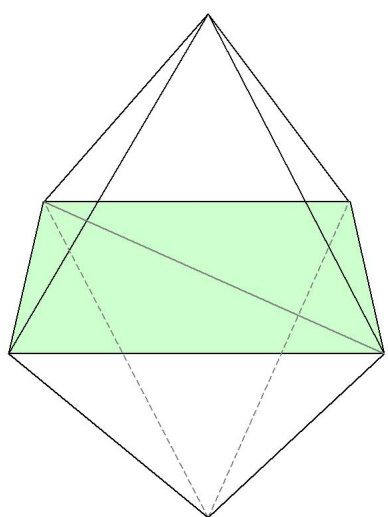

Fig. 9. Starting point for incremental insertion of topographic features: two 'air' tetrahedrons and two 'earth' tetrahedrons. Note that these initial tetrahedrons will contain the entire topographic model and thus are extremely large.

\subsection{Proposed Implementation}

The new modelling approach is still triangulation-based, as one still wants to benefit from the well-defined character and its strong computational and analytical capabilities. As a result the whole topographic model will be stored as one large TEN. The triangulation and tetrahedronization of newly inserted objects can be performed separately in order to improve performance; the resulting edges can be inserted into the TEN after the initial triangulation/tetrahedronization. In order to maintain a volume partition one does not start with an empty model, but with four initial tetrahedrons, see Figure 9 These four tetrahedrons (two 'air' tetrahedrons and two 'earth' tetrahedrons) will ensure that all space between features is modelled to without the need for explicitly surveying and maintaining 'air' or 'earth' objects. Note that in order to enclose the complete model these initial tetrahedrons are very large, as in theory a complete country or more can be modelled. The process of modelling topographic features consist of four discernable steps. 
1. Start with four initial tetrahedrons, two 'air' and two 'earth' tetrahedrons;

2. Refine the earth's surface by inserting height information from a DEM;

3. Refine 'air' and 'earth' tetrahedrons in case of ill-shaped tetrahedrons by insertion of Steiner points;

4. Add real topographic features;

Within this last step both non-volumetric and volumetric features might be triangulated or tetrahedronized separately using a constrained triangulation/tetrahedronization algorithm. The outcome of these algorithms can be inserted incrementally in the full topographic model. As mentioned earlier there are no algorithms for the construction of constrained TENs capable of handling constrained faces. As a result one needs to 'translate' a constrained face into a set of constrained edges to preserve this constrained face in a tetrahedronization. This is done by first triangulating the boundary faces of an object and inserting all resulting edges as constrained edges into the tetrahedronization. As a postprocessing step one needs to label the boundary faces explicitly as constrained faces.

Triangulating or tetrahedronizing the features one-by-one before insertion in the topographic model reduces computational complexity and thus saves computer time. The results need to be inserted into the full topographic model. This requires the use of an incremental algorithm to avoid recomputing the whole model. As the complete topographic model (the TEN) will be stored in a spatial database, it is necessary to implement the incremental algorithm within the database. As a result a full DBMS approach is required, instead of using the database just to store results of the computations.
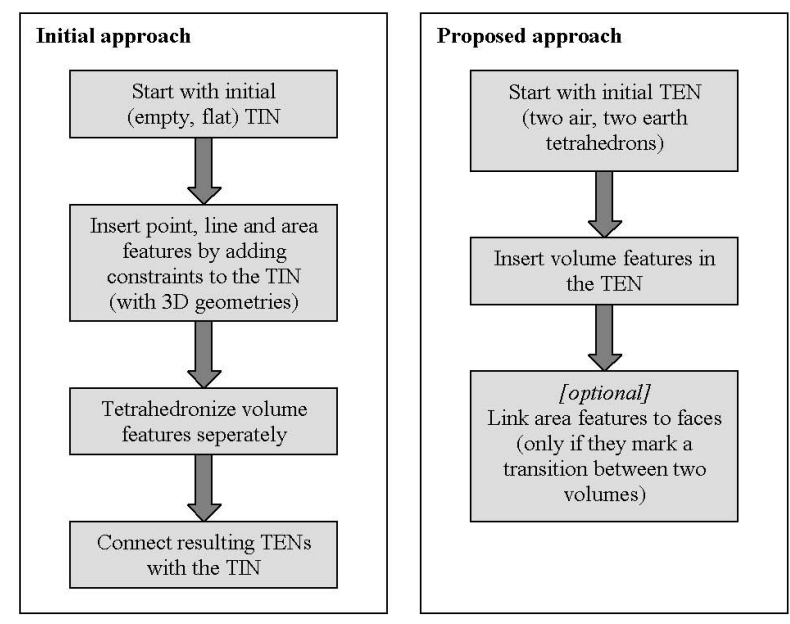

Fig. 10. Summary of the two proposed approaches 


\section{Conclusions and Future Research}

This paper first introduced a very pragmatic approach of $3 \mathrm{D}$ modelling as it aims at modelling as much as possible in (less complicated) 2.5D, where full 3D modelling will be applied only in exceptional cases. Triangulations were selected as data structure due to their strong computational capabilities. The triangulation and tetrahedronizations can be integrated at a conceptual level, as both TIN and TEN use nodes, edges and faces. However the actual connection at design level appeared to be very difficult. As a result a more rigid approach was designed to solve the problems. Both approaches are summarized in 10. An important assumption is that the $3 \mathrm{D}$ model should form a volume partition and that preferably features are modelled in $3 \mathrm{D}$. If one wants for some reason to use a less dimensional representation, this should only be done when these less dimensional features mark the transition between two 3D objects. For objects located on the earth's surface this is very clear: these objects might be modelled as faces instead of volumes, because one of the neighbouring volumes will represent 'air' and the other 'earth'.

As the rigid concept shows great promise, the following topics need further research:

1. Develop an UML class diagram of the rigid model

2. Implement required algorithms for constrained triangulation and tetrahedronization

3. Implement within the DBMS an incremental algorithm for tetrahedronization

4. Perform real tests with real world features

5. Define views within the TEN to offer TIN functionality on the earths surface

\section{References}

Carlson, E. (1987), Three-dimensional conceptual modeling of subsurface structures, in 'Auto-Carto 8', pp. 336-345.

de Cambray, B. (1993), Three-dimensional (3D) modelling in a geographical database, in 'Auto-Carto 11', pp. 338-347.

Kolbe, T., Gröger, G. \& Plümer, L. (2005), Citygml: Interoperable access to 3d city models, in 'Geo-information for Disaster Management (GI4DM)', Springer, pp. 884-899.

Molenaar, M. (1990a), A formal data structure for three dimensional vector maps, in '4th International Symposium on Spatial Data Handling, Zürich', International Geographical Union IGU, Columbus, OH, pp. 830-843.

Molenaar, M. (1990b), A formal data structure for three dimensional vector maps, in 'Proceedings First European Conference on GIS (EGIS'90, Volume 2', Amsterdam, pp. $770-781$.

Molenaar, M. (1992), 'A topology for 3D vector maps', ITC Journal 2, 25-33.

Pilouk, M. (1996), Integrated Modelling for 3D GIS, PhD thesis, ITC Enschede, Netherlands.

Schönhardt (1928), 'Über die Zerlegung von Dreieckspolyedern in Tetraeder', Mathematische Annalen 98, 309-312. 
Shewchuk, J. R. (1997), Delaunay refinement mesh generation, PhD thesis, Carnegie Mellon University.

Shewchuk, J. R. (2004), 'General-Dimensional Constrained Delaunay and Constrained Regular Triangulations I: Combinatorial Properties', To appear in: Discrete 86 Computational Geometry. Available at http://www-2.cs.cmu.edu/ jrs.

Stoter, J. (2004), 3D Cadastre, PhD thesis, Delft University of Technology.

Zlatanova, S. (2000), 3D GIS for urban development, PhD thesis, Graz University of Technology. 